\title{
Quality of Service and Service Provisioning on a Competitive Market
}

\author{
Lambert J.M. Nieuwenhuis $^{1}$ and Ing Widya ${ }^{2}$ \\ ${ }^{1}$ KPN Research, the Netherlands \\ ${ }^{2}$ University of Twente, the Netherlands \\ L.J.M.Nieuwenhuis@kpn.com, widya@cs.utwente.nl
}

\begin{abstract}
The objective of this paper is to provide an economic and commercial context for QoS research in open distributed environments. The analyses are based on a telecommunications value chain model. The model is used to define possible roles for telecommunications companies, migrating from traditional telephony network operators towards privatised ICT companies in a new economy. Based on these developments, we advocate $\mathrm{R} \& \mathrm{D}$ activities aiming at standardised concepts and engineering principles to establish and control QoS in open distributed environments.
\end{abstract}

\section{Introduction}

At the beginning of the $21^{\text {st }}$ century, developments on the telecommunications services market are far from stable. The process of privatisation of the incumbent operators has not been completed yet. Almost every day, we read articles in newspapers on the world's largest mergers and acquisitions, which primarily were related to the communication industry, and recently also include the content industry. On the other hand, at national and European level, we are confronted with new regulations, e.g., preventing that companies dominate a national market. Besides these developments, the Internet has grown explosively and now governs today's developments in many aspects. It is not surprising that the Internet has a major influence on our economy on the telecommunications industry and on our ICT technologies. In this paper, we will discuss a number of these developments from a network operator's perspective in more detail to obtain a context for the technological challenges that face the service provisioning industry in the years to come. We believe that in a mature and open market, as in many other industries, the primary economic forces will be determined by customers selecting from a wide range of services and products that differ with respect to price and quality.

In the years to come, the quality of service will not primarily be determined by the available bandwidth of our networks anymore. Quality of Service (QoS) will more and more be determined by the availability of all the resources needed for service provisioning, e.g., the communication links, the routers, the computing devices, and data stores. From the major trends and developments from a network operator's perspective we derive research questions that in our view should be addressed by the distributed computing community in the years to come. 
The paper is structured as follows. In Section 2, we present our telecommunications value chain model, involving multiple players in our industry. In Section 3, we compare the added value of various players in these value chains. In Section 4, we elaborate on Internet service provisioning as it is today. In Section 5, we identify new players and roles in the telecommunications value chain. In Section 6, we discuss how the industry can benefit from standards and concepts for establishing and control of QoS in an open distributed telecommunications environment. We summarise our findings in Section 7.

\section{Telecommunications Value Chain Model}

For many years, developments in the field of telecommunications research have been driven by an isolated and autonomous joint effort of telecommunications manufacturers and network operators. They developed standards co-ordinated by dejure and industrial standardisation organisations comprising both manufacturers and network operators. In sharp contrast with this, Internet technologies are emerging very

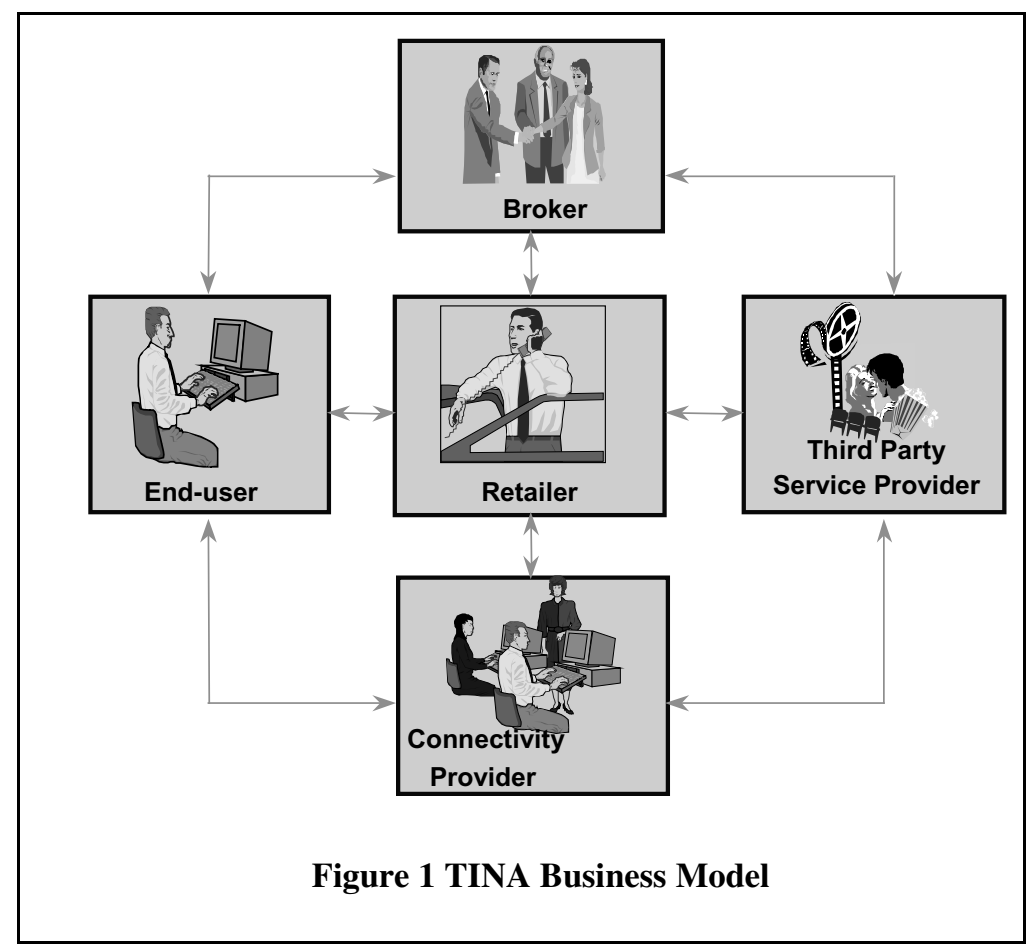

rapidly and in an open, collaborative, and distributed process, with many participants, originally dominated by the academic world. The Internet is now governing the entire telecommunications industry. Hence, Internet will be the basis for a universal service 


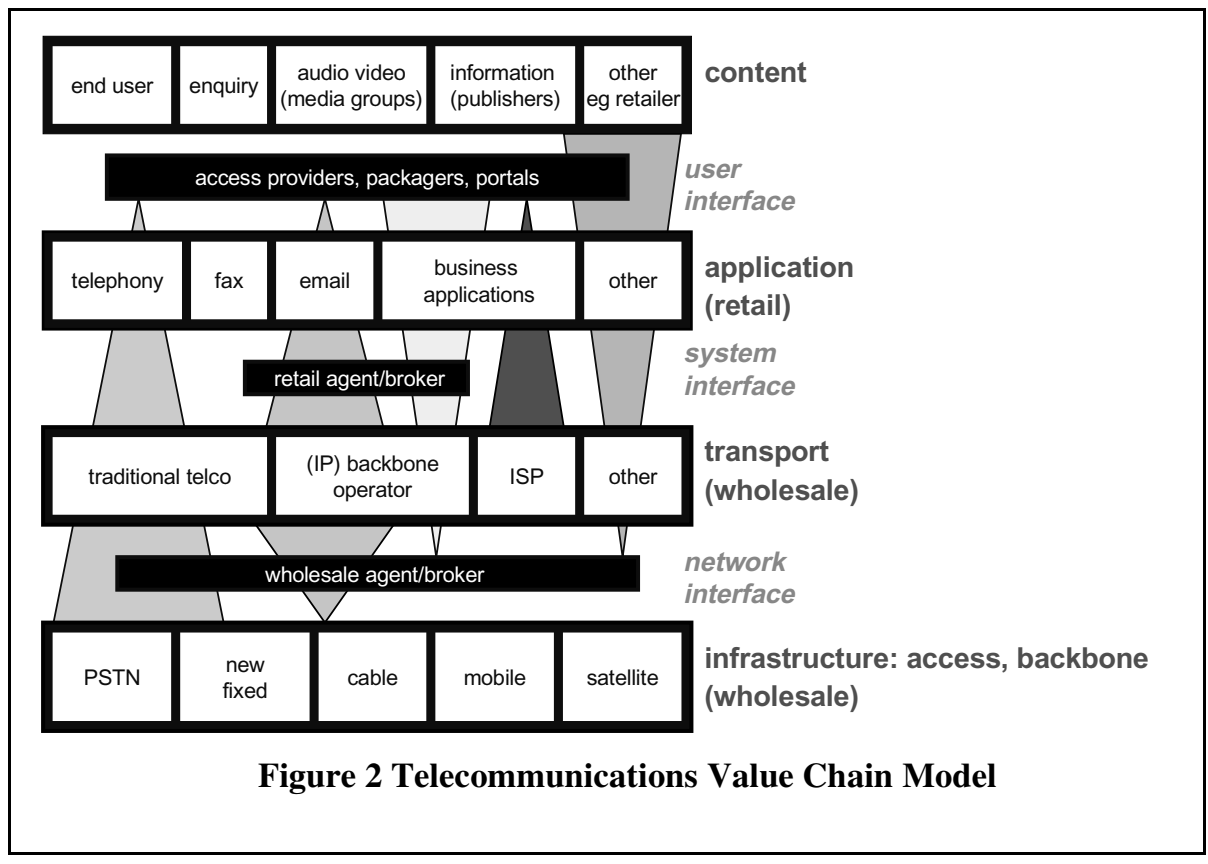

market. The Internet will continue to develop, which requires large-scale, massmarket technologies, fitted to serve the needs of the open market. Figure 1 shows the TINA Business Model [1], emerged from developments that started more than five years ago in the telecom industry. The power is that TINA has shown the world that a business model should be the basis for technological developments. The model is however very generic. We believe that in practice, a more detailed and dynamic model is needed to understand the roles, stakeholders and companies of tomorrow's world.

In Figure 2, we present a picture of our model that describe the developments in the telecommunications industry in Europe. The model represents the value chains of both, the 'traditional' telecommunication and media content industries, as well as the newly emerging Internet industries. This model in fact depicts various value chains, which can be constructed using elements from four layers, i.e., content, applications, transport, and infrastructures.

Content can be multimedia data generated by end users, in case of one-to-one or one-to-many communication. Content may be any type of multimedia data, e.g., enquiry, advertisement, e-commerce information, news, traffic or stock exchange information, and multimedia data related to entertainment. Applications are tailored end-user services, which encode content into a form suitable for transport. Traditionally, we see telephony and fax services, but also Internet services like email and web, which will develop into more complex business applications needed for various E-commerce applications. The transport layer encompasses all transmission and routing of packaged and encoded content. Finally, fixed and wireless networks are needed to carry the information across the world. 
The model also depicts the various roles that need to be played in the value chains of the industry. We distinguish between end-users, providing their own content for communications purposes and commercial content providers. There are retailers, maintaining the relationship with customers and providing applications offerings to the end-users. For example, an end-user subscribes to a mobile telephony service that is used to transfer content from one end-user to the other. At the transport level, we find wholesale transport companies, e.g., traditional telecommunications companies, selling voice network capacity or data modem pools. Finally, at the bottom of our model, we find the network operators, exploiting fixed, wireless and satellite networks, e.g., the plain old telephony networks, cable networks, mobile telephony and data networks and various types of satellite networks.

Currently, the market place is developing. Basically, we see two major developments. First, we see traditional telecommunications companies on the move from governmental organisations towards privatised companies present at the stock markets. Second, we are at the beginning of the IP revolution, i.e., we are heading for a world where virtually everybody and everything is on line. It means that new roles in the value chain will emerge. Content packagers or portals collaborate with several content providers, creating a position to offer customers a broad range of information and other content. In between the application and transport layers, the technical function of connecting applications and transport services is being combined with a commercial function. Retail brokers or retail agents are linking different applications and different transport services. They offer customers a broad range of applications and transport services, provided by others. Transport and the actual physical infrastructure are sometimes linked through wholesale agents or wholesale brokers, buying and selling (backbone) network capacity.

\section{Generating Added Value}

In the previous section, we have presented a telecommunications value chain model. In fact, all parties are needed in order to deliver a service to the end-user. Economical and technological forces suggest that parties in the top rows of the model add more value than parties in the bottom rows. In order to determine which roles in the model are more favourable, it is relevant to determine who has direct access to the customers. We can distinguish between physical, commercial and perceptual access to the customers.

- Physical access to the customer is a crucial factor. For the residential market, owning the public telephony network is an extremely important asset. Currently, narrow band technologies like PSTN and ISDN are currently provided. In Europe, broadband technologies like ADSL are currently being rolled out. Cable networks extent their original TV distribution services with telephony and IP services. They also create added value from their physical access to residential customers. Additionally, wireless and satellite networks play an increasingly important role. In the business markets, newcomers start connecting customers with own networks, in order to decrease their dependency on national, public network operators. A strong position could be obtained, when a company includes one or more elements of each layer of the telecommunications value chain model shown in Figure 2. 
- Commercial access to customers seems to be even of more importance than having physical access. Companies that posses this type of access set the brand in the market, determines the customer interface and can tailor the customer interface to provide marketing information of other players in the value chain.

- Finally, perceptual access to customers relates to the chase of eyeballs. The number of people that can be reached through various media creates value. We generally want to maximise the number of people actually reading newspapers and magazines, watching programs on TV channels, or hitting Web pages on the net. The number of eyeballs directly relates to the way players make money in the telecommunications value chain.

In general, a position at the top of the telecommunication value chain model (see Figure 2) is more favourable than a position on the bottom. Perceptual access can be obtained through interesting and preferable unique content. Applications create value for end-users and create a commercial relationship. Transport and infrastructures are anonymous. It is difficult to generate more revenues than cost price with a fair margin. As infrastructures become cheaper and cheaper, the margins will decrease proportionally.

\section{Internet Service Provisioning}

In this section, as an example, we elaborate the model that is currently being used in Europe to provide Internet services to end-users. We show that it is complicated from both, the end-user's perspective and an economic perspective.

Figure 3 shows a picture of the current Internet model, which for a comparison can be related to Figure 1, the TINA model. In general we need to subscribe to a service to obtain a PSTN or ISDN line for setting up a connection to an Internet Service Provider (ISP). This service is in most cases provided by a traditional telephony company. These types of connections can also be set-up through a mobile telephony company using on GSM or GPRS and in the future UMTS. An IP dial tone can be obtained from an ISP. In Europe some ISPs provide these and other basic Internet services for free, others demand subscription fees. The Internet services are used to access content of a content provider. Currently, much content on the Internet can be accessed for free. A limited set of content can only be accessed after paying a certain amount of money, either in the form of a subscription fee or on a pay per view basis. In a number of cases, security support or payment services require yet another party. Hence, for current Internet service provisioning multiple roles need to be fulfilled. In the current situation different companies fulfil these roles. Consequently, the consumers and end-users have to deal with a number of different companies. In order to improve the Customer Relationship Management (CRM), we may expect that retailers and brokers will provide end-users with one stop shopping concepts. Obviously, this puts requirements on the technologies that are needed to actually make this happen.

One of the most challenging developments with Internet service provisioning relates to the business model. Using Figure 3, we also see which companies may send a bill to users of Internet service providers. First, a public network operator can send a bill for setting up a connection between the end-user and the ISP. In Europe, in most 


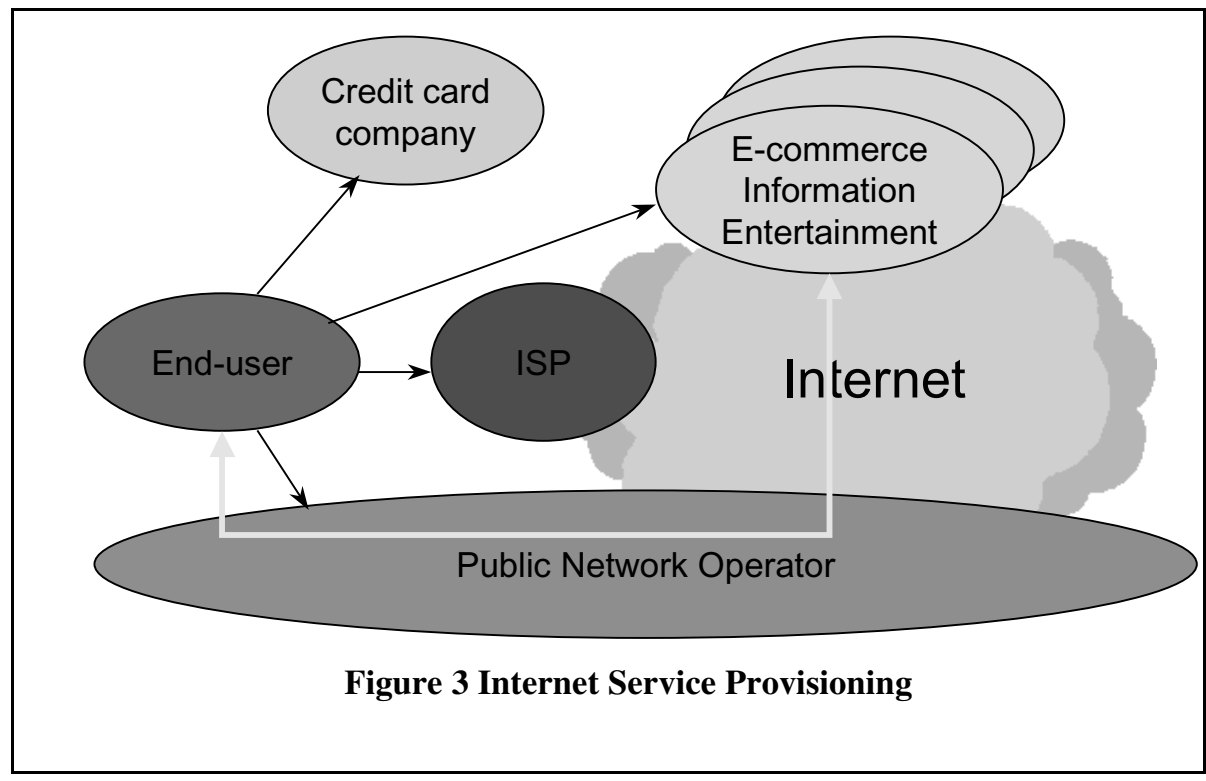

cases users are charged on a connect time basis. In the US, usually users pay a flat fee, i.e., they are charged independent of the connect time. Second, ISPs charge users for the services they provide, e.g., mail and web hosting, and the interconnection to the Internet. Various models exist. Due to increased competition, we see a rapidly growing number of ISPs that provide their services for free. In Europe, ISPs make traffic revenue-sharing agreements with public network operators in order to survive. It is unclear, whether regulators in Europe will allow this type of interconnections between ISPs and public network operators. Besides this type of revenue sharing with network operators, ISPs expect to generate revenues from advertisements and revenue sharing models in E-commerce transactions. It is still unclear, whether these business models are strong enough to let ISPs survive.

As the number of ISPs is growing, in both the residential and business segments, as in any other industry, Quality of Service (QoS) starts to become a critical success factor in the ISP business. In the past, ISPs have been unable to offer QoS agreements as because of problems with infrastructure reliability and availability. In the business segment, customer attitudes to QoS are changing, because IP connections are becoming a key factor for delivering business applications, and users are increasingly dissatisfied with the 'best effort' level of service that ISPs provide today.

In this section, we have shown that Internet Service Provisioning is maturing. Consequently, we expect that such issues as customer care, costs and revenues, and Quality of Service will play an increasingly important role.

\section{Open Services Market}

Currently, in the middle of the year 2000, the telecommunications industry is in a process of what is called unbundling. Traditional telecommunications companies are 
followed by investors and compared to their competitors. The stock market demands transparency of telecom operators, and rewards increased transparency with a higher share price. At the same time we see new companies entering the market place, playing only a few or even a single role in our telecommunications value chain model. Definitely, unbundling enables these companies to become a specialist in their element of the value chain. On the other hand, for users and customers, the service provisioning becomes more complicated, as we have seen in our Internet Service Provisioning example in the previous section. Therefore, we may expect that brokers will play more and more important role in telecommunications value chain model. Examples of brokers are content packagers, retail and wholesale brokers. A growing customer base will strengthen their position in the telecommunications value chain.

In fact, the vertical and horizontal unbundling in the telecommunications value chain model creates a number of interesting win-win positions. A content provider benefits if the number of outlets increase. An application provider benefits if more content becomes available from a variety of content providers. An ISP benefits from an increase of applications, and a network operator benefits from more traffic on its network. In fact, this was also the idea behind the $3^{\text {rd }}$ party service-provisioning role as indicated by the TINA business model (see Figure 1).

Obviously, the co-operation between different parties in the market needs to be enabled by technological developments. Standards are needed. The development of standards has undergone a major change. The IETF and OMG and a number of other similar organisations have shown how fast standards can be developed compared to the traditional bodies like ITU-T and ISO. However, what remained the same through the years are the huge commercial interests related to standardization. Strong players in the market try to establish and keep market shares through de-facto standardisation of their products.

At this place, we would like to underline the major differences and commonalties between such telecommunications standardisation activities as TINA and other developments like IETF, W3C, or OMG.

Due to our experience gained with TINA [11], we believe that the Service Architecture concepts are a valid vehicle to obtain standards for services that are much more complicated than the services we encounter today on the Internet. Setting up a multimedia, multiparty, multi-domain service, which guarantees secure and reliable connections in a closed user group, definitely requires some standards. It is also likely, that such high quality connections can only be provided when users are prepared to pay for it. We also believe that such open standards for distributed object technologies as CORBA can provide the infrastructure to create such open services market.

\section{Quality of Service Research Activities}

In previous sections, we have presented the telecommunications value chain model and discussed some of the commercial forces that determine the developments in the telecommunications arena. The development of an unbundled open services market implies more competition with multiple players in various areas. As the market matures, we believe that a variety of product-market combinations will create 
differentiation on basis of Quality of Service (QoS) in particular and Service Level Agreements (SLAs) in general. In this section, we propose to combine the results of the telecommunications value chain model and integrate the need for standards, open services market, and the need to control the end-user QoS.

The notion of QoS is very broad and applies to many areas of service provisioning. The bottom line is of course the QoS as perceived by the end-user. However, the perceived QoS depends on the QoS of the constituting components of the system that deliver the service. Accordingly, the QoS is determined by the QoS of the content, the application, the transport systems and the underlying networks. From a technological point of view, we believe that the end-user perceived QoS of multimedia services is determined by the way distributed systems are capable of handling such things as availability and reliability of bandwidth, jitter, delay of audio and video signals. But, from an end-user perspective, we have to translate QoS in user oriented terms to increase their awareness of the delivered QoS.

There are typically two ways to have a distributed system deliver a certain QoS. One is to over-dimension the system to easily support peak usage. This approach is widely used in practice, in particular in the Internet. However, over-dimensioning is generally inefficient and often unattractive because a lot of system capacity remains unused when the system is lightly loaded. In general, the system usage is determined by at least three factors, which are nondeterministic in practice:

- The number of users that need to be served at a certain point in time

- The amount of resources that each user needs at a certain point in time

- The number of faults disturbing the system at a certain point in time

The alternative approach to deliver QoS is to dimension the system more conservatively and make system's components QoS aware. This means that the system has a notion of the QoS of information streams and distributed processing, and that it can use its resources accordingly. These resources comprise not only network elements but also the software infrastructure, e.g., the web servers in today's systems and multimedia servers capable of storage and retrieval of audio and video information.

There are a number of QoS standardisation and research activities going on in organisations like ISO, IETF, ITU-T, TINA, OMG and various research groups, like GMD Fokus in Berlin, COMET group at Columbia University, Distributed Multimedia Research Group in Lancaster. Overviews of the activities of these groups can be found in [2], [3], [4].

At the University of Twente, we participate in several projects of the Telematics Institute on QoS research. In the AMIDST project [5], we focus on end-to-end QoS control in open distributed environments, like for example CORBA. We believe that QoS control is also needed for the software infrastructures that are used to control QoS of underlying networks and of processes that stream multimedia information. We search for a generic QoS control framework for establishment of QoS, for both operational and multimedia streaming control loops, and for which a diversity of QoS control mechanisms can be developed, for example, mechanisms for QoS (re)negotiation, QoS computation, QoS monitoring and QoS re-enforcing. In fact we would like to complement CORBA's functional specifications with QoS specifications, and generate code for QoS control [6], [7], [8].[9], [10]. We are currently focussing our research on the following issues: 
- A challenging question is how to relate the end-user QoS to the QoS of the subsystems, constituting the entire system. This includes investigation on QoS specification, mapping of QoS characteristics to capability of underlying resources in local sites and mechanisms for peer to peer mapping and control of QoS, both in the establishment and operational phases of services[6].

- We are considering telematics services, engineered in such open distributed object environments as CORBA. The challenge is to establish and control the QoS of these telematics services at the level of the distributed environment rather than the applications itself. In this way, we can design and develop the telematics services independent of applications. We looking for CORBA extensions to create QoS awareness. This research also relates to current activities within the $\mathrm{OMG}$ on replication and fault tolerance. Here, we look at the management of component and object based systems.

- In the field of audio and video streams, we try to overcome heterogeneity in a multi provider environment (e.g. an environment with fixed and mobile network providers). We are investigating solutions for which dynamically connected piecewise homogeneous parts model heterogeneity. Topics that are in study are, for example, mechanisms to support multimedia scaling and transcoding, mechanisms for smooth (i.e. QoS preserving) hand-over when end-users move across homogeneous parts or providers management domains. We also look at the influence of SLAs between the end-user and provider but also between the providers on the choice of previously mentioned mechanism.

\section{Conclusions}

In this paper, we have presented a telecommunications value chain model. We have used this model to describe how horizontal and vertical unbundling progresses in the telecommunications industry. We have shown that the telecommunications industry is developing towards a real open and competitive market. QoS provisioning delivers an import contribution to these developments. We have identified a number of interesting subjects for further research, focusing on concepts and engineering principles on the establishment and control of QoS in distributed open environments. 


\section{References}

[1] TINA Business Model and Reference Points, Version 4.0, May 1997, TINA C Deliverable

[2] C. Aurrecoechea, T. Campbell, and L. Hauw, A Survey of QoS Architectures 1998.Center for Telecommunications Research Columbia University.

[3] De Meer, J. and Hadif, A. "The Enterprise of $Q o S " .98$.

[4] F.A. Aagesen, QoS Frameworks for open distributed processing systems, Telektronik, vol. 1.97, no. magazine on Quality of Service in Telecommunications, pp. 26-41, 1997.

[5] AMIDST project, http://amidst.ctit.utwente.nl/

[6] C. Hesselman, I. Widya, A.T. van Halteren, L.J.M. Nieuwenhuis Middleware support for media streaming establishment, accepted for IDMS2000, October 2000

[7] M. Wegdam, D.J. Plas, A.T. van Halteren, L.J.M. Nieuwenhuis, ORB Instrumentation for Management of Corba, accepted for PDPTA2000, Las Vegas, June 26-29, 2000

[8] L. Bergmans, A.T. van Halteren, L. Pires, M. van Sinderen, M. Aksit, A QoS-Control Architecture for Object Middleware, accepted for IDMS2000, October 2000

[9] M. Wegdam, A.T. van Halteren, Experiences with CORBA interceptors, Position paper for the Workshop on Reflective Middleware (RM 2000), co-located with the IFIP/ACM International Conference on Distributed Systems Platforms and Open Distributed Processing (Middleware2000), April 2000

[10] A.T. van Halteren, A. Noutash, L.J.M. Nieuwenhuis, M. Wegdam, Extending CORBA with specialised protocols for QoS provisioning, Proceedings of International Symposium on Distributed Objects and Applications (DOA'99), September 1999.

[11] L. J.M. Nieuwenhuis, A. T. van Halteren, EURESCOM Services Platform, Proceedings of Telecommunications Information Networking Architecture Conference 1999 (TINA '99), Apr. 1999.

\section{About the Speaker Lambert J.M. Nieuwenhuis}

In 1980, Lambert J.M. Nieuwenhuis joined KPN Research, the innovation centre of KPN in The Netherlands. His interest in the area of Information and Communication Technology includes fault tolerant, parallel, and distributed computing. Within KPN Research, he had several positions including project manager, secientific advisor and Head of Strategy. He was also leader of European projects on distributed object technologies. Currently, Lambert J.M. Nieuwenhuis is heading the department of Middleware. He is also parttime professor at the Computer Science and Electrical Engineering Faculty of the University of Twente. He has a BSc and MSc in electrical engineering and $\mathrm{ahD}$ in Computer Science. 